\title{
Collaborative Logical Framework: An e-Learning assesment tool in .LRN platform
}

\author{
*Jose Vega Alvarado, Agustín Francesa Alfaro ${ }^{\dagger}$, Mario Chacón Rivas ${ }^{\ddagger}$ y César Garita Rodríguez ${ }^{\S}$ \\ TEC Digital, Instituto Tecnológico de Costa Rica \\ Cartago, Costa Rica \\ Correo: $\left\{{ }^{*}\right.$ jvega,${ }^{\dagger}$ afrancesa,${ }^{\ddagger}$ machacon,${ }^{\S}$ cesar $\} @$ tec.ac.cr
}

\begin{abstract}
In the teaching and learning process, one of the most widely used assessments under the constructivist model is to work in groups. This has many advantages, but also weaknesses such as lack of collaboration, poor organization, difficulty in assessing, poor communication, among others. This paper describes the functionality of the Collaborative Logical Framework and the redesign made by the TEC Digital Tecnológico de Costa Rica (TEC) which aims to aid students and teachers to reduce the problems that can be generated working in groups, through a tool integrated in a LMS applying usability criteria on web and mobile platforms and some integrations with others applications on .LRN LMS. This was validated applying a survey to grade and postgrade students at the TEC. The results were favorable, such as the usability of the interface, in a (very low) scale of 1 to 5 (very high) average usability by the twenty seven students surveyed is 4 points.
\end{abstract}

Keywords-e-learning, collaborative logical framework, software, usability, dotLRN, responsive

\section{Introducción}

Como hace referencia Santos [1], el Marco Lógico es "una metodología utilizada por las Agencias de Cooperación Internacional para gestión de proyectos, ya que ayuda a aclarar los objetivos de cualquier proyecto al facilitar la identificación de las relaciones de causalidad previstas y determinar los riesgos que podrían impedir el logro de los objetivos del mismo. Con él se consigue implicar a las partes interesadas para que intervengan en el proceso de planificación y seguimiento".

Para realizar alguna actividad grupal de manera efectiva, se debe proporcionar, según Costaguta [2] los medios necesarios para incentivar a los participantes a trabajar de forma colaborativa. Con el fin de resolver las dificultades como la mala comunicación, abandono, falta de participación y evaluación que se generan en una actividad grupal; se crea la herramienta Marco Lógico Colaborativo (MLC), la cual busca facilitar la colaboración mediante el uso de preguntas, expresión de opiniones y evaluación del conocimiento adquirido, estudiando las características colaborativas que tiene cada persona en un grupo de trabajo.

Tener una herramienta que proporcione las facilidades de colaboración para el usuario no es el único objetivo del
MLC, sino también brindar a las personas que la usan una forma fácil, intuitiva y diseñada con el objetivo de facilitar su uso en una población que prefiere ingresar a este tipo de herramientas desde dispositivos móviles que desde una computadora [3]. Según estudios hechos por el periódico El Financiero $^{1}$, en Costa Rica cada vez más personas ingresan a navegar desde celulares, tabletas, poniendo en evidencia la importancia de implementar un diseño responsive a los usuarios del MLC.

En estudios anteriores realizados en Madrid [4] donde se probó el MLC en los laboratorios de la UNED de España, se logró reunir datos para obtener resultados que mostraron problemas en cuanto a la percepción de facilidad de uso, ya que la mayoría de tutores y estudiantes que lo utilizaron, no lograron completar todo el proceso.

Sin embargo, gracias a los análisis anteriores se logra crear escenarios eficaces que permiten el aprendizaje a través de la interacción, exploración, discusión y la construcción de aprendizaje colaborativo.

Es por esto que el Marco Lógico Colaborativo es diseñado mediante el uso de la metodología de diseño de interfaces en TEC Digital del Instituto Tecnológico de Costa Rica (TEC). La cual busca eliminar las dificultades que se presentaron en la versión anterior del MLC, que se mencionarán en la sección de antecedentes de este artículo (ver sección 2).

Este artículo se compone de las siguientes secciones: Antecedentes, trabajo relacionado, Marco Lógico Colaborativo, metodología del rediseño, evaluación y resultados, limitaciones, Conclusiones y Trabajo futuro.

A continuación se describen los antecedentes del MLC.

\section{Antecedentes}

El primer MLC que se puso a prueba en un escenario real fue realizado en el año 2009, durante semana de la ciencia en un colegio de Madrid España cuyo objetivo fue obtener las percepciones del MLC y los indicadores de colaboración propuestos.

Estos estudios fueron realizados por estudiantes entre catorce y diecisiete años de edad por una mecánica conocida

1. http://www.elfinancierocr.com/tecnologia/Red 506-celular-Internet-cibernautas-navegacion-redes_sociales_0_590940940. html 
como gymkhana ("Rally") el cual consistía en tres etapas de veinticinco minutos cada una, donde los estudiantes debían escribir de manera colaborativa una historia hablando sobre tres elementos científicos dados: uno científico, un invento $\mathrm{y}$ un lugar.

Como resultado del análisis de la prueba, se logra mostrar que el enfoque del MLC permite la creación de escenarios que aseguran la colaboración y aprendizaje de los estudiantes mediante la retroalimentación de los miembros del grupo (comunicación, discusión, auto-evaluación y acuerdo) [4].

Además, el sistema fue capaz de obtener métricas de interacción entre el estudiante y el sistema. El prototipo proveía herramientas para la configuración de las métricas, reglas y la definición de los indicadores obtenidos, sin embrago durante el análisis de los estudios pudieron observar que algunos de los indicadores se traslapaban con la definición de otros, por lo que se agruparon aquellos indicadores que tenían un significado similar.

Más adelante, tras tres años del primer estudio del MLC, se realiza un nuevo estudio [4] con el objetivo de obtener mejores resultados, este fue realizado en un laboratorio de aDeNu donde se prepararon cuatro puestos con la infraestructura para reunir los datos emocionales con diferentes sensores que permiten grabaciones fisiológicas (electrocardiogramas, la respuesta galvánica de la piel, la frecuencia respiratoria y la temperatura corporal) y grabaciones de comportamiento (registros del teclado, ratón y cara).

Para el diseño del estudio, se tomó en cuenta trabajos recientes en la computación que sugieren el uso de un enfoque multi-modal combinando diferentes fuentes de datos emocionales pueden mejorar los resultados obtenidos. Con estos antecedentes mencionados se construyó la secuencia de interacciones.

El proceso de realización del MLC fue realizado mediante varios pasos que aseguran una buena extracción de los datos necesarios para las actividades colaborativas en un ambiente web; uno de estos pasos fue llenar una encuesta al final de cada fase del MLC (individual, colaborativa, consenso) conocida como SAM (Self Assessment Manikin) donde los participantes debían informar acerca de sus sentimientos al realizar la tarea colaborativa del MLC como por ejemplo "Cuando hice esta tarea me sentí....", “Cuando hice esta tarea he pensado en ....".

Al finalizar la prueba se explicó a los participantes los objetivos de la investigación y los participantes contaron de una forma más informal acerca de su experiencia con la herramienta del MLC.

Como resultado de estos estudios, se analizó los datos obtenidos de la encuesta SAM, dicha información mostró que la etapa de colaboración del MLC parece ser más atractiva y dinámica mientras que no fue así para la etapa de consenso. Según a los investigadores de aDeNu esto se debe a que los estudiantes hicieron todo el proceso completo por lo que cuando llegaron a la etapa de consenso tuvieron que esperar a que el moderador de cada grupo propusiera la respuesta de acuerdo.
En cuanto a la percepción de la facilidad de uso de la herramienta por parte de los estudiantes los datos demostraron que hay ciertos problemas que mejorar ya que los resultados obtenidos a partir de los once participantes de los tres MLC analizados eran más bien baja (promedio: 56.43 de cada 100; desviación estándar: 13,45).

Por lo tanto, una calificación promedio de sesenta y ocho ha sido extraída de más de quinientos estudios, lo que significa un puntaje bajo según el Sistema de Escala de Usabilidad (SUS por sus siglas en inglés) sesenta y ocho se considera por debajo del promedio. Los problemas de usabilidad más críticos que requieren mejoras son reducir los pasos para llevar a cabo ciertas acciones y para hacer más claro al participante cómo acceder a las etapas de colaboración y consenso.

Además con respecto a los indicadores de colaboración obtenidos durante la prueba, se pudieron obtener ocho participantes intuitivos, ocho participantes útiles y seis participativos, estos indicadores se explicarán en la sección de descripción de la herramienta.

A partir de estos estudios del grupo aDeNu sobre el aprendizaje colaborativo en un entorno web, el TEC Digital implementa una nueva interfaz para el MLC donde se tomaron en cuenta los aspectos de usabilidad, mencionados en la sección de metodología del rediseño, los cuales son necesarios para brindar a los estudiantes que usan la herramienta una forma fácil, dinámica y sobre todo que facilite la colaboración en los grupos de trabajo combatiendo los problemas que las primeras dos pruebas realizadas.

En la siguiente sección se describirá la herramienta y las facilidades que ofrece la herramienta previo al rediseño hecho por el TEC Digital.

\section{Trabajo relacionado}

Según Scagnoli [5], el concepto de aprendizaje colaborativo ha sido objeto de investigación y estudio en los últimos años con la aparición del e-learning. El término "aprendizaje colaborativo" hace referencia a metodologías de trabajo que impulsan al grupo a cooperar hacia el logro de un mismo objetivo.

Scagnoli nos comenta que la educación a distancia sobre entornos virtuales facilita la exploración y búsqueda individual de información y conocimiento, permiten al estudiante reforzar sus habilidades en la investigación y construcción del propio aprendizaje. El uso de aprendizaje colaborativo obliga al profesor a pasar del rol de informante principal y centro de conocimiento, a facilitador del aprendizaje. El estudiante en una actividad colaborativa deja de ser un receptor pasivo convirtiéndose en el principal actor de su propio conocimiento en la interacción con materiales y con sus pares.

Además, el autor indica que normalmente las herramientas utilizadas por el docente para asignar un trabajo colaborativo, son aquellas que permiten la comunicación y colaboración entre individuos independientemente de su ubicación geográfica, evitando la utilización de un software especial. A continuación se describirá, algunas de las herramientas 
utilizadas para cumplir con el objetivo de fomentar un buen aprendizaje por medio de la cooperación:

- Foros de discusión o debate: Es un medio de comunicación entre miembros de una comunidad virtual. En los cursos observados en la investigación de la Universidad de Illinois en Estados Unidos [5] los foros se usaban como centros de debate, discusión, y pedido de información. Los temas tratados estaban relacionados con los temas de la clase o con preguntas de índole metodológica o técnica. Además pudieron observar que los participantes recibían retroalimentación y conocimiento del aporte individual de cada uno de los miembros construyendo conclusiones bien informadas. Algunos foros eran moderados por el docente, expertos, o por líderes de grupos, otros eran libres.

- Blogs: Técnica utilizada con el fin de compartir o recolectar información, donde una persona puede crear un espacio personal en la Web de manera fácil, sin necesidad de conocer de diseño Web.

- Wikis: El uso de Wikipedia, Wikibooks, WikEd y Wiki en Moodle ofrecía aprendizaje colaborativo que se daba por la construcción de documentos sobre diversos temas entre los participantes.

- Grupos Virtuales: Son espacios en Internet adonde las personas pueden crear libremente una membresía y luego crear su comunidad virtual para compartir archivos, información, y para comunicarse sincrónica o asíncronamente.

- Listas de distribución: Es un conjunto de correos electrónicos utilizados para compartir información y expresar opiniones entre los miembros que la conforman.

Con el uso de estas herramientas como apoyo para la cooperación, es importante comentar las técnicas/actividades usadas para fomentar un buen aprendizaje, por medio de la colaboración de los miembros. Según estas investigaciones, las actividades que consumieron más interacción por parte de los estudiantes es la asignación de en un proyecto o propuesta común, donde se observó que los participantes del grupo motivaban el aprendizaje y tenían un efecto de conjunto provocando una "interdependencia positiva" [5].

Este tipo de actividades fueron realizados por medio de foros y blogs para la comunicación y el intercambio de ideas. Otra técnica que destacó es la de realizar revisiones o críticas sobre el trabajo realizado por los compañeros, en donde los estudiantes mostraban sus trabajos escritos a un compañero y este lo analizaba devolviéndolo con sus comentarios al autor. El objetivo de esta actividad era promocionar la interacción, motivando el pensamiento crítico mediante la lectura de trabajos similares y educar en la fundamentación de comentarios de texto, de esta manera los integrantes del grupo se ayudaban unos a otros para trabajar eficiente y efectivamente, mediante la contribución individual de cada miembro [5].

Una vez terminada todas las actividades se asignó a cada grupo evaluador de otro grupo, esto incentivó el compromiso y la práctica de habilidades en la comunicación y resolución constructiva de conflictos con otros miembros de la clase. El uso de chats, foros, wikis e intercambio de archivos facilitó este tipo de actividades. Estas herramientas y actividades, usadas para fomentar el aprendizaje colaborativo representan un aporte que facilita el uso de la cooperación tanto para los docentes que asignan este tipo de técnicas para el aprendizaje, como también para los estudiantes que las usan para aprender [5]. En el MLC se proporciona a ambos actores el conjunto de estas herramientas y técnicas usadas en una sola herramienta, mejorando la cooperación, comunicación y aprendizaje de aquellos que la usen.

\section{Marco Lógico Colaborativo}

La herramienta MLC pretende facilitar actividades grupales tanto para los profesores que las crean y revisan, como para los estudiantes que la realizan, incentivando que trabajen de forma conjunta, aprendan y realicen de manera apropiada la tarea.

Por su parte, el MLC da inicio cuando el profesor asigna una tarea al estudiante usando el MLC, durante este proceso el profesor brindará la información necesaria para la creación de una actividad grupal usando la herramienta.

Una vez que el MLC fue creado el estudiante deberá realizar las actividades propuestas por el profesor mediante tres fases, este proceso ofrecerá los medios necesarios para que la colaboración sea efectiva, la cual según Salomon [6], se obtiene si hay interdependencia genuina entre los estudiantes que están colaborando. Esa dependencia genuina se describe como:

1) Compartir información para generar discusión y nuevas conclusiones.

2) Generar roles complementarios para la distribución de las tareas.

3) Compartir los conocimientos de forma clara y detallada.

Como lo muestra la Figura 1, las fases son conocidas como: individual, colaborativa y consenso. Cada una de ellas tienen un objetivo específico el cual se explica a continuación:

- Fase Individual: En esta fase los estudiantes trabajan de forma independiente a los demás compañeros de grupo. El objetivo de esta fase es garantizar que todos los miembros de cada grupo hagan su propio trabajo y evitar cargas de trabajo a un solo estudiante. Además el estudiante tiene la facilidad de escribir en un foro sus dudas y consultas, las cuales el profesor podrá solventar. Por medio de esta fase el estudiante crea su primera versión la cual será compartida en la fase colaborativa con sus compañeros de trabajo.

- Fase Colaborativa: El objetivo principal de esta fase es incentivar la colaboración aprendizaje y retroalimentación entre los miembros de cada grupo de trabajo, para así crear mejores versiones del trabajo 
realizado previamente tomando en cuenta la retroalimentación obtenida por sus compañeros. Para lograr este objetivo, el estudiante podrá comentar y calificar las respuestas hechas por sus compañeros en la fase individual y colaborativa.

- Fase Consenso: Su propósito es llegar a un acuerdo realizando una versión final que será presentada al profesor, esta última versión solo puede crearse y editarse por el estudiante moderador de cada grupo de trabajo. El estudiante moderador es aquel estudiante que obtenga los indicadores de colaboración más altos (participativo, comunicativo y útil) o bien sí son elegidos por el profesor.

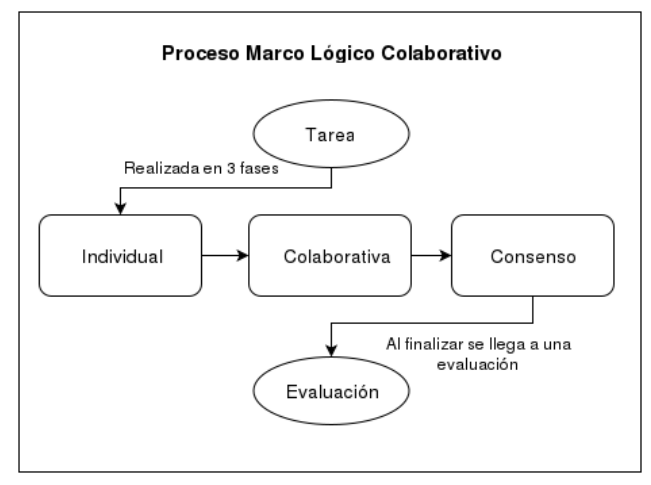

Figure 1. Proceso MLC.

La versión que el moderador proponga como solución final, podrá ser comentada y calificada por el resto de compañeros no-moderadores realizando un "consenso", para así llegar a obtener la mejor versión posible y ser presentada al profesor.

Durante la realización del MLC los estudiantes generarán indicadores de colaboración según sea su comportamiento en cada una de las fases. Estos indicadores se dividen en dos tipos de indicadores: los activos y los pasivos.

Los indicadores activos son aquellos que hacen referencia a interacciones que se almacenan en la base de datos como por ejemplo foros en los que ha participado, número de versiones creadas, número de comentarios hechos y número de puntuaciones hechas durante el desarrollo del MLC. Todos estos datos guardados en la base de datos son usados por las métricas activas de cada indicador activo (participativo, perspicaz, útil, no-colaborativo, con iniciativa, comunicativo). Los indicadores activos son calculados por ahora mediante conteos de las acciones almacenadas por el estudiante durante la actividad colaborativa.

La definición de cada indicador activo para el MLC según Santos [1] son los siguientes:

- Participativo: Mide la actividad que tiene el estudiante durante el trabajo.

- Perspicaz: Persona que tiene participación en los mejores aportes del trabajo.

- Útil: Estudiante que ayuda a sus compañeros a mejorar sus aportes.
- No-colaborativo: Persona que no tiene actividad colaborativa con sus compañeros.

- Con iniciativa: Estudiante que realiza acciones por su propia motivación.

- Comunicativo: Alumno que comparte información con sus compañeros.

Ahora bien los indicadores pasivos son todas aquellas iteraciones entre el sistema y el estudiante, éstas son registradas con ayuda de una herramienta llamada Tracking and Auditing Module (TAM) [7], donde cada uno de los registros guardados formarán las métricas que construyen los indicadores pasivos del MLC. Estos indicadores se calculan mediante un análisis del flujo de eventos, por ejemplo, que hizo un estudiante después de abrir un foro.

A continuación se describirá el significado de cada indicador pasivo del MLC según menciona Santos [1]:

- Cotilla: No tiene clara la tarea por lo que se busca en las respuestas de los demás.

- Inspirable: Se basa en los aportes de sus compañeros para crear el su aporte.

- Inspirador: Sus aportes son considerados importantes, y son referenciados por sus compañeros.

- Pensativo: Persona que antes de colaborar analiza los aportes de sus compañeros.

- Concienzudo: Lee muchas respuestas y mensajes antes de hacer su aporte.

- Inseguro: Alumno que revisa constantemente su trabajo, lo edita y crea muchas versiones.

Los indicadores activos y pasivos construirán un perfil de cada estudiante el cual es de gran interés para la evaluación de una actividad de este tipo. Es importante aclarar que los datos guardados en la herramienta TAM no son solo datos generados por el MLC, sino que esta herramienta se encarga de registrar todos los movimientos que un usuario realiza sobre la plataforma del TEC Digital.

Como es de esperar al almacenar en TAM tanta cantidad de acciones realizadas por los usuarios en la plataforma, se observó que las consultas que realizaba el MLC para calcular las métricas, tomaban mucho tiempo en responder, por lo que se optimizó cada consulta desarrollada para disminuir la cantidad de tiempo de consulta y así ofrecer más flexibilidad a los usuarios que consultaban este tipo de información.

El MLC también brinda facilidades al profesor que lo utiliza ya que gracias a esta herramienta, se puede llevar un seguimiento individual y grupal de cada uno de los estudiantes.

Además, el profesor tiene la facilidad de aclarar dudas y generar sugerencias que los estudiantes puedan tener durante su realización, (una de las desventajas principales que se le presenta al profesor es el evaluar una actividad grupal) es por esto que se facilita el seguimiento del proceso de los estudiantes que participan en un MLC, brindándole información relevante sobre cuál estudiante realizó un trabajo apropiado y quién no. 


\section{Metodología del rediseño}

Antes de describir el rediseño realizado, se comentan los objetivos que busca la nueva versión del MLC, mencionadas a continuación:

1) Disminuir la complejidad y cantidad de pasos.

2) Mejorar la navegación y re-direccionamiento de las páginas al usuario, para evitar confusiones.

3) Evitar pasos independientes para realizar una acción.

4) Ofrecer a sus usuarios un claro panorama del estado de la tarea y la fase en la que se encuentra.

5) Evitar que el usuario ligue pasos, si no que el sistema se encargue de manejar automáticamente un hilo conductor de los pasos del MLC.

6) Ofrecer al profesor un panorama global de la tarea colaborativa.

7) Facilitar la comprensión del flujo del proceso del MLC.

8) Ofrecer a los usuarios la posibilidad de ingresar a la herramienta desde cualquier dispositivo, mediante la implementación de una interfaz responsive.

Para cumplir los objetivos mencionados anteriormente, el rediseño de la interfaz se realizó bajo atributos de usabilidad mencionados por Nielsen [8] de la siguiente manera:

- Fácil de aprender: El sistema debe de ser fácil de aprender para que el usuario pueda iniciar lo más rápido posible a usarlo.

- Eficiencia: Se debe facilitar un alto nivel de productividad una vez que se aprende a usar herramienta.

- Fácil de recordar: Debe de ser sencilla de recordar, con el fin de que un usuario ausente pueda ser capaz de usarla sin necesidad de volver a aprender.

- Errores: Se debe tener bajo nivel de errores, para que el usuario comenta el mínimo de ellos y si los comete que tenga la oportunidad de recuperarse de ellos.

- Satisfacción: La herramienta debe de ser agradable de usar, para que los usuarios queden satisfechos cuando la usen.

A través de los años la usabilidad según Nielsen [9] se ha vuelto más valiosa y presente en la economía de internet, la cual es de gran importancia ya que una página web sin usabilidad representa pérdidas a nivel de visitas y costos, un usuario que no se sienta atraído a una herramienta, simplemente busca otra que si le satisfaga sus necesidades.

Teniendo en cuenta los objetivos de esta versión, se implementa la metodología del rediseño la cual consta de tres etapas (Investigación y Análisis, Planeamiento y Desarrollo de la propuesta y Diseño gráfico de la interfaz) que se representan en la Figura 2.

Etapa 1-Investigación y análisis:

En esta etapa, se analiza la usabilidad de la herramienta y se realiza un listado de los contenidos que posee, con esto se busca representar de una forma más clara el alcance de todo el contenido que conforma el MLC. Además se

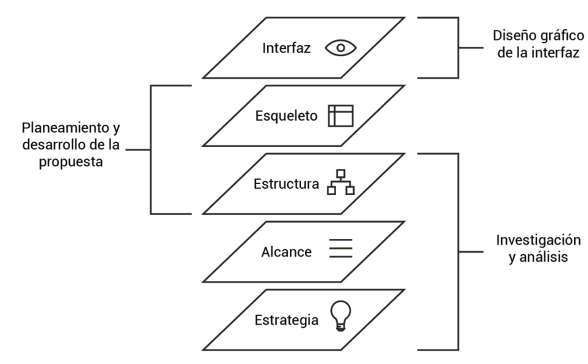

Figure 2. Metodología del Rediseño

ubica el contenido por medio de la jerarquía del contenido y por medio del URL, cuando se ubica el contenido se identifica el tipo de contenido (caja de selección, caja de texto, selectores, botones, entre otros).

Etapa 2-Planeamiento y desarrollo de la propuesta:

En esta etapa se realiza la arquitectura de la interfaz donde se representa los contenidos que posee y los relaciona entre ellos, evidenciando los pasos que el usuario debe realizar para llevar a cabo una acción específica. Luego de definir la arquitectura se utiliza una herramienta conocida como Card Sorting la cual ayuda a mejorar el etiquetado, la agrupación y la organización de la información [10]. Posteriormente se interpreta y se compara las categorías realizadas por el usuario con las que se definieron en la arquitectura con el fin de que todo sea coherente.

Etapa 3-Diseño gráfico de la interfaz:

Para el diseño de la interfaz del MLC se utiliza las metodologías como Moodboard, Look \& fell y Animatic.

A continuación se describe su implementación y sus resultados.

\subsection{Implementación}

Después de analizar los hallazgos encontrados en las primeras pruebas del Marco Lógico Colaborativo se busca la manera de contrarrestar los problemas encontrados y se implementa el rediseño de la interfaz gráfica, con el fin de que sea más amigable y fácil de usar, para promover una colaboración más efectiva.

Teniendo en mente la problemática existente el MLC fue modificado bajo un objetivo claro que consistía en crear una interfaz fácil de entender, evitar la gran cantidad de pasos para crear una evaluación de MLC y la disponibilidad de uso de la herramienta en cualquier dispositivo.

Para el desarrollado se utiliza el lenguaje TCL ('Tool Command Language") en la plataforma .LRN/OpenACS, usando HTML5, CSS3, Bosstrap, Jquery, Librería Estándar del TEC Digital ${ }^{2}$ y PostgreSQL como base de datos.

El primero de los cambios en la interfaz fue la funcionalidad de crear un MLC, ya que la interfaz anterior

2. http://tecdigital.tec.ac.cr/tds-lib/\#/ 
provocaba que los profesores que usaban esta herramienta se perdieran y no completaran todo el proceso necesario, esto se debía a que el profesor tenía que completar una gran cantidad de pasos y navegación sin guía para completar la creación del MLC. Debido a ese problema se creó una guía paso a paso ("Wizard"), que brinda al profesor instrucciones más claras y concisas. A continuación se describen los siete pasos para crear un MLC:

Paso 1: Elegir el curso donde se agregará el nuevo Marco Lógico Colaborativo, se presenta la lista de cursos que el profesor imparte.

Paso 2: Solicita al profesor un nombre y una descripción para el MLC.

Paso 3: El profesor deberá definir las instrucciones que el estudiante debe conocer de cada fase y las fechas límite de cada una de ellas, este paso facilita la asignación de fechas y horas ya que no permite al profesor elegir una fecha anterior a la actual, además al definir una de las fechas limita inmediatamente a la próxima.

Paso 4: Elegir el documento que se asignará a la tarea creada previamente, donde se especifica el trabajo que los estudiantes deben de realizar.

Paso 5: Realizar los grupos respectivos. En este paso el profesor tiene la facilidad de definir cuantas personas quiere por grupo, creando los necesarios para la cantidad de personas definidas por el profesor. Otra de las funcionalidades importantes de este paso, es la opción de definir los grupos de trabajo de manera aleatoria o bien definirlos manualmente mediante un "Drag and Drop" al grupo que se desea.

Paso 6: El profesor escoge si el moderador se elegirá de manera automática o manual, en la manera automática el sistema se encargará de la elección del moderador de cada grupo según los indicadores que los estudiantes obtengan durante la fase individual y colaborativa. Mientras que de forma manual el profesor debe elegir a un estudiante por grupo.

Paso 7: El profesor establece el valor que tendrá la evaluación dentro del curso.

Uno de los requerimientos necesarios por parte de las funcionalidades del profesor, es el seguimiento de cada uno de sus estudiantes a través de las fases del MLC, así podrá ver quien participa y ayuda a sus compañeros. Para cumplir con este requerimiento se crea una interfaz que ofrece al profesor la posibilidad de ver las respuestas de sus estudiantes, realizar sus propias recomendaciones y aclarar dudas por medio de comentarios a través de un foro.

Cuando se termina el proceso, se muestra al profesor la opción de ver los trabajos finales presentados por cada grupo de trabajo o bien la indicación de que no se presentó el documento final, junto con las funcionalidades de calificar el trabajo realizado de manera individual o grupal.

Además, el profesor tiene la opción de ver las fotos de los estudiantes para así facilitar la identificación de cada uno de ellos cuando se califica. Una característica importante en la evaluación de un estudiante, es que el profesor posee una mayor cantidad de insumos para realizar la calificación, por medio de información relevante como los indicadores de colaboración que el estudiante adquirió durante el proceso del MLC y la respectiva cantidad de métricas que el estudiante cumplió para poder formar dicho indicador.

Por otro lado, la interfaz del estudiante también fue modificada ya que existían ciertas complicaciones al entrar a la fase colaborativa y consenso; para resolver este problema se integraron todas las fases, es decir, en una sola página se muestra lo correspondiente a cada fase según las fechas límites indicadas por el profesor. A continuación se describe cada uno de los elementos que comprenden las fases del MLC para el estudiante:

Interfaz Individual:

El estudiante que se encuentra en la fase individual tiene la facilidad de descargar la tarea asignada por el profesor, crear una versión, editarla, comentarla y publicarla. Además siempre está visible el ícono de perfil de usuario y ayuda, para que revise el estado de sus indicadores en su perfil o bien para recibir ayuda correspondiente a la fase.

Interfaz Colaborativa:

Las facilidades que ofrece esta interfaz son desarrolladas con el objetivo de incentivar a los estudiantes a comunicarse mediante el uso de foros en donde cada uno de ellos escribe las dudas y sugerencias, y así fomentar la colaboración entre los participantes del grupo. Además el estudiante calificará las respuestas de sus compañeros para incentivar a los estudiantes para realizar un buen trabajo.

\section{Interfaz Consenso:}

Esta interfaz está desarrollada para dos tipos de estudiantes, el moderador y el no-moderador. El estudiante moderador puede ver las versiones creadas por sus compañeros y los comentarios de cada uno de ellos, para crear la versión final y publicarla para recibir retroalimentación. Por otra parte el estudiante no-moderador tiene la facilidad de comentar y calificar las versiones publicadas por el moderador del grupo, pero no de crear nuevas versiones.

Posteriormente al rediseño del MLC se espera contrarrestar la dificultades de los estudiantes al realizar las actividades propuestas por el profesor e incentivanr a la comunicación y colaboración. Con la utilización de las guías paso a paso, tooltips e instrucciones se espera que los profesores usen la herramienta del MLC como solución a los trabajos, proyectos, tareas que se realizarán de manera grupal.

A continuación se mencionará la integración del MLC con otros módulos del TEC Digital, para ofrecer más beneficios al profesor y estudiante. 


\subsection{Integraciones a la herramienta}

A continuación se describen las integraciones de esta herramienta con otros módulos del TEC Digital.

\subsubsection{Integración módulo Evaluaciones y Rúbricas.} Cualquier profesor que implemente una metodología de enseñanza colaborativa se puede plantear la siguiente pregunta ¿Hasta qué punto se si mis alumnos están trabajando colaborativamente?, ¿Sabemos con certeza que se crea una interdependencia positiva entre los miembros del grupo y que se comparta información para el apoyo mutuo entre los miembros de una actividad colaborativa? Y aún más ¿Cómo puedo evaluar el trabajo hecho colaborativamente? [11].

Teniendo en cuenta estas preguntas, se integra el módulo de evaluaciones y rúbricas al MLC con para poder realizar la actividad colaborativa como una evaluación del curso al cual se asoció durante su creación, junto con las funcionalidades que una tarea normal posee desde el módulo de evaluaciones, así como, el poder crear una nueva, ver la evaluación creada, editarla y calificarla.

La opción de calificar una actividad colaborativa del MLC es la que contiene más cambios ya que ofrece más ventajas a los profesores, ofreciendo información del proceso del MLC desde la evaluación, brindando más insumos al profesor cuando realiza la calificación respectiva. La información que se muestra en la evaluación de un MLC es el documento final que entregó cada grupo, la opción de mostrar los indicadores que obtuvo cada estudiante junto con su respectiva descripción y cantidad de métricas que cumplió el usuario para adquirir dicho indicador. Además se muestra la última nota obtenida por el estudiante en la fase colaborativa del MLC. Estas características que se mencionan buscan resolver las dudas que se pueden generar por parte del profesor cuando califica el MLC.

Como apoyo a dicha evaluación, el MLC tiene la opción de poder usar una rúbrica. En este modelo, se busca medir, según Ally [12], los diferentes niveles de calidad para una tarea específica.

Por lo tanto, el uso de las rúbricas es de gran importancia para el uso de herramientas de evaluación, ya que mejoran el desempeño del estudiante propiciando condiciones favorables para el aprendizaje, puesto que el estudiante entiende los criterios de evaluación deseados por el profesor al realizar su trabajo.

Además cuando los profesores evalúan los trabajos, proyectos, investigaciones, MLC, entre otros, saben con claridad qué determina la calidad de dichos productos. La mayoría de los educadores que utilizan la herramienta de rúbricas encuentran que la evaluación se vuelve un proceso más objetivo y que les permite brindar retroalimentación más precisa al estudiante sobre aspectos que podría mejorar.

Teniendo estas dos herramientas integradas, hace que el MLC sea una herramienta más completa brindando más apoyo a la evaluación colaborativa para el profesor.
5.2.2. Integración con el módulo de Control de Plagio. Control de Plagio es un módulo del TEC Digital, el cual ofrece al usuario una herramienta para medir el nivel de similitud que tiene un documento que es comparado con una base de datos de texto exactos y completos a nivel mundial. Este módulo es agregado a las funcionalidades del profesor y el estudiante, con el fin de ofrecerles la posibilidad de conocer la validez del documento que se crea y entrega como solución final.

5.2.3. Integración con el módulo de Notificaciones. La integración con la herramienta de Notificaciones pretende facilitar tanto profesores como estudiantes, dándoles aviso de ciertos eventos que se generan durante el proceso de la actividad, esta integración comprende notificaciones como:

- Creación de la primera versión en la fase individual, la cual notifica al profesor, para que el educador esté informado de que hay algo que revisar, sin necesidad de ingresar a la herramienta.

- Creación de versiones durante la fase colaborativa, tanto para el profesor como para los estudiantes que forman parte del grupo.

- Creación de versión de consenso, la cual notifica al profesor y a los estudiantes no-moderadores del grupo de trabajo.

- Nuevo comentario en algún foro, para los miembros del grupo al cual se le comentó.

- Se notifica al estudiante cuando el profesor realizó la evaluación respectiva.

Gracias a estas notificaciones se pretende tener a los participantes de una evaluación tipo MLC lo más informados posible sobre todas las actividades que pueden suceder durante todo el proceso de colaboración. En la Figura 3 podemos observar un ejemplo de las notificaciones a los participantes. Seguidamente se presenta la sección de evaluación y resultados.

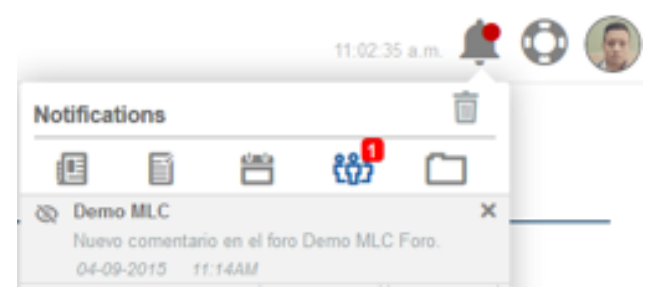

Figure 3. Notificaciones para MLC

\section{Evaluación y Resultados}

Para conocer los resultados de la herramienta MLC sobre todo en la percepción de uso para los usuarios y los problemas que trata de resolver esta aplicación, se implementó una encuesta para profesores y estudiantes, que pretende analizar los resultados que se obtuvieron a manera de plan piloto en tres cursos en el Tecnológico de Costa Rica. A 
continuación se detallaran los resultados de la encuesta ${ }^{3}$ aplicada en dichos cursos desde la perspectiva del profesor:

- Un $100 \%$ de los profesores consideran que la facilidad de creación de un MLC es intermedia.

- El seguimiento de los estudiantes durante la actividad colaborativa y su evaluación fue un poco más difícil de usar, sin embargo pudieron observar, participar de la actividad y hacer su respectiva evaluación.

- Un $66,7 \%$ de los profesores que ingresaron a la actividad desde un dispositivo móvil pudieron realizar las funcionalidades con normalidad, el resto no acceso desde un dispositivo móvil.

- Todos los profesores opinan que el uso de las notificaciones mejora de manera significativa el involucramiento de los estudiantes con el trabajo.

- Según a las respuestas dadas los profesores piensan que el uso de foros en el MLC facilitó y fortaleció en buena medida $(66,7 \%)$ la comunicación entre los estudiantes, así como entre el profesor y los estudiantes.

- El $100 \%$ de los profesores piensan que metodología utilizada por la herramienta MLC favorece el trabajo colaborativo entre los estudiantes.

- En cuanto a los indicadores de colaboración, estos fueron de gran valor para la evaluación en un 33,3\% de los encuestados, el otro $66,7 \%$ casi no los tomo en cuenta en la evaluación.

- El seguimiento de los estudiantes durante todo el proceso fue el principal insumo en la evaluación del trabajo en un $66,7 \%$ de los encuestados, el resto lo consideraron en menor medida.

- El MLC ayudó a conocer a un $66,7 \%$ de los encuestados el trabajo que realizo cada uno de sus estudiantes.

- En cuanto a los criterios que más se fomentaron en la actividad según los profesores podemos mencionar la comunicación $100 \%$, trabajo en equipo $33,3 \%$, trabajo colaborativo $100 \%$, mejor coordinación $33,3 \%$, carga equitativa del trabajo $66,7 \%$, facilidad al evaluar $100 \%$, participación de los estudiantes $100 \%$ y evitar separación de los miembros del grupo 33,3\%.

Ahora se menciona algunas de las respuestas que los estudiantes respondieron en la encuesta ${ }^{4}$ :

- Como podemos observar en la figura 4 , un $48,1 \%$ de los estudiantes se le facilitó el uso de la herramienta desde sus dispositivos móviles, un $25,9 \%$ se le dificultó pero pudo realizar lo que quería, un 3,7\% tuvo problemas al usarlo y el resto simplemente no acceso a la actividad desde un dispositivo móvil.

- La mayoría de los estudiantes $(55,5 \%)$ opinan que el uso de notificaciones influyó en muy buena medida su desempeño en el trabajo.

3. https://drive.google.com/file/d/0B-aHNHuJ_du6clNfSnNVTnRZSE0/ view?usp=sharing

4. https://drive.google.com/file/d/0B-aHNHuJ_du6VU5pT1RFTjJGVkk/ view? usp=sharing
- En cuanto al uso de foros estos fortalecieron y facilitaron la comunicación entre sus compañeros y con el profesor donde 20 estudiantes $(74,1 \%)$ de los 27 encuestados respondieron positivamente.

- Los indicadores de colaboración fueron de mucha motivación a mejorar las estrategias de colaboración en el trabajo a un 70,3\% de los encuestados.

- En cuanto a los criterios que más se fomentaron en la actividad según los estudiantes podemos mencionar la comunicación $(66,7 \%)$, trabajo en equipo $(66,7 \%)$, trabajo colaborativo $(74,1 \%)$, mejor coordinación $(29,6 \%)$, carga equitativa del trabajo $(48,1 \%)$, participación de los compañeros $(77,8 \%)$ y evitar la separación de miembros del grupo $(29,6 \%)$.

- Los estudiantes opinaron que las fases que comprenden el MLC(individual, colaborativa y consenso) les permitió entender fácilmente el flujo de la actividad. Donde un $74,1 \%$ de los encuestados contestaron positivamente.

- La usabilidad (calidad de la experiencia que tiene un usuario cuando interactúa con un producto o sistema) de la herramienta fue medida entre alta y muy alta, en la fase individual $51,8 \%$, colaborativa $74,1 \%$ (figura 5) y consenso $52,8 \%$.
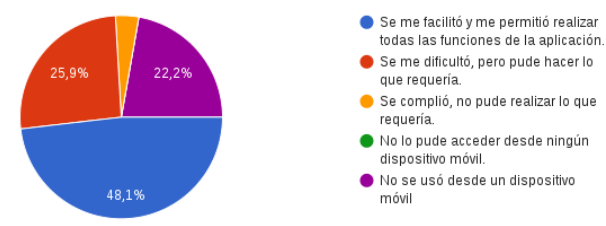

Figure 4. Estadísticas responsive

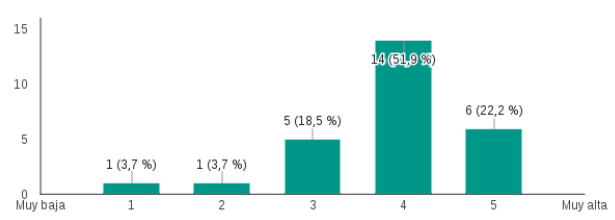

Figure 5. Estadísticas usabilidad fase colaborativa

\section{Limitaciones}

En esta sección, se presentan las limitaciones relacionadas al trabajo realizado:

- Con respecto a la muestra en la encuesta, se utilizó el cien por ciento de los cursos actuales que cuentan con la herramienta; esto debido a que el proyecto finalizó su fase de desarrollo a finales del 2015 y antes de ser público para toda la universidad, se realiza una fase de pruebas piloto, para luego realizar la puesta en producción del mismo junto a la capacitación respectiva, previsto a inicios del 
segundo semestre del 2016. Por lo tanto, la encuesta es realizada únicamente en los tres cursos del plan piloto de pruebas.

- Esta herramienta actualmente se encuentra disponible en la plataforma dotLRN, utilizada tanto en la UNED de España como en el Tec Digital del Tecnológico de Costa Rica.

\section{Conclusiones y Trabajo Futuro}

Las principales conclusiones de este trabajo son:

- La importancia de una buena interfaz es relevante ya que de esta manera se garantiza el uso de la herramienta, muchas veces se tiene una muy buena solución a nivel de funcionalidades pero la interfaz no es agradable al usuario final y no se usa.

- La importancia de una herramienta responsive es muy importante para garantizar la accesibilidad desde cualquier dispositivo, ya que la mayoría de las personas ingresan a los sitios web desde un dispositivo móvil (celular, tableta, entre otros) como lo podemos observar en las encuesta realizada.

- La herramienta del MLC reduce el tiempo que los estudiantes invierten en organizar un trabajo grupal ya que pueden realizar su trabajo grupal desde cualquier lugar. Además reciben retroalimentación de los demás compañeros de grupo y de esta manera se incentivan a realizar un mejor desempeño y así obtener indicadores de colaboración que reflejen el trabajo realizado produciendo aprendizaje colaborativo.

- El uso de foros es de gran relevancia en el MLC como se ve reflejado en la encuesta aplicada a los estudiantes, ya que es el canal por el cual cada integrante expresa sus opiniones y más importante aún, es el me dio por el cual se genera el aprendizaje asertivo entre los miembros de un MLC.

- Una de las grandes ventajas que ofrece el MLC es el uso de rúbricas para la evaluación de la tarea, ya que estas brindan beneficios tanto al profesor como al estudiante, facilitando al estudiante el poder analizar los criterios que el profesor usará para evaluar.

- El uso de notificaciones brinda información actualizada sobre las actividades realizadas por los compañeros en un MLC, esto es importante ya que mantiene al estudiante involucrado en la actividad.

Como trabajo futuro se implementará la integración con el módulo de GAAP (Gestor de Actividades de Aprendizaje), de esta forma se pretende utilizar las actividades de aprendizaje con la metodología del MLC y se buscará una mejor forma de procesar los datos que forman los indicadores de colaboración, con el fin de brindar indicadores más precisos a los conceptos que los definen.

Además, con la puesta en producción de la herramienta, así como el inicio de las capacitaciones, se pretende realizar de nuevo la encuesta, así como también pruebas de eye- tracking, para tener resultados más específicos que puedan indicar si es necesario realizar algunos ajustes a la propuesta.

\section{References}

[1] O. C. Santos, A. Rodríguez, E. Gaudioso, and J. G. Boticario, "Cómo gestionar la colaboración en el Marco Lógico Colaborativo en un entorno de aprendizaje adaptativo basado en web," Inteligencia Artificial, Revista Iberoamericana de Inteligencia Artificial, vol. 24, pp. 121-129, 2004. [Online]. Available: http://aepia.lcc.uma.es/index.php/ia/article/view/786/778

[2] R. Costaguta and E. Durán, "Group and students profiles to support collaborative learning in a multiagent model," in Advances in New Technologies, Interactive Interfaces and Communicability. Springer, 2012, pp. 134-139. [Online]. Available: http://link.springer.com/ chapter/10.1007/978-3-642-34010-9_13

[3] "IEEE Xplore Abstract - Implementing responsive web design for enhanced web presence." [Online]. Available: http://ieeexplore.ieee.org/Xplore/defdeny.jsp?url=http\%3A\% 2F\%2Fieeexplore.ieee.org\%2Fstamp\%2Fstamp.jsp\%3Ftp\%3D\% 26arnumber\%3D6596440\%26userType\%3Dinst\&denyReason= $-134 \&$ arnumber=6596440\&productsMatched=null\&userType=inst

[4] O. C. Santos and J. G. Boticario, "Involving Users to Improve the Collaborative Logical Framework," The Scientific World Journal, vol. 2014, pp. 1-15, 2014. [Online]. Available: http: //www.hindawi.com/journals/tswj/2014/893525/

[5] N. I. Scagnoli, "Estrategias para motivar el aprendizaje colaborativo en cursos a distancia," 2005. [Online]. Available: https://www.ideals. illinois.edu/handle/2142/10681

[6] "What does the design of effective CSCL require and how do we study its effects?" [Online]. Available: http://dl.acm.org/citation.cfm? id $=130909$

[7] J. Couchet, O. C. Santos, E. Raffenne, J. G. Boticario, and D. Manrique, "A General Tracking and Auditing Architecture for the OpenACS framework," Departamento de Investigación y Desarrollo Universidad Galileo, p. 179, 2008. [Online]. Available: https://www.researchgate.net/profile/Jesus_G_Boticario/publication/ 229022918_A_General_Tracking_and_Auditing_Architecture_for_ the_OpenACS_framework/links/00b495295c5405e645000000.pdf\# page $=181$

[8] "Usability Engineering - Jakob Nielsen - Google Libri." [Online]. Available: https://books.google.it/books?id=95As2OF67f0C\& printsec $=$ frontcover\&hl $=i t \&$ source $=g b s \_g e \_s u m m a r y \_r \& c a d=0 \# v=$ onepage $\& \mathrm{q} \& \mathrm{f}=$ false

[9] "Web usability - Jakob Nielsen - Google Libri." [Online] Available: $\quad$ https://books.google.it/books?id=IY3Lprpb2qgC\& printsec $=$ frontcover\&hl=it\&source=gbs_ge_summary_r\&cad=0\#v= onepage $\& \mathrm{q} \& \mathrm{f}=$ false

[10] D. E. Zimmerman and C. Akerelrea, "A group card sorting methodology for developing informational web sites," in Professional Communication Conference, 2002. IPCC 2002. Proceedings. IEEE International. IEEE, 2002, pp. 437-445. [Online]. Available: http://ieeexplore.ieee.org/xpls/abs_all.jsp?arnumber=1049127

[11] A. I. Cuéllar and M. I. Alonso, “ ¿ Cómo afrontar la evaluación del aprendizaje colaborativo? Una propuesta valorando el proceso, el contenido y el producto de la actividad grupal/How evaluate the collaborative learning? A proposal valuing the process, content and the product of group interactivity," Revista General de Información y Documentación, vol. 20, p. 221, 2010. [Online]. Available: http://search.proquest.com/openview/ dd09b90b1c8a5a526108d543e3342c75/1?pq-origsite=gscholar

[12] H. G. Andrade, "Using rubrics to promote thinking and learning," Educational Leadership, vol. 57, no. 5, pp. 13-19, 2000. [Online]. Available: http://www-tc.pbs.org/teacherline/courses/ rdla230/docs/session_2_andrade.pdf 